INPLASY

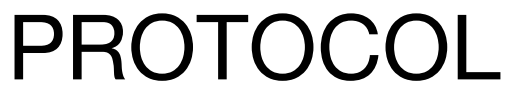

To cite: Prada et al. Overview of systematic reviews and meta-analyses published in Portuguese Medical journals: time trends and critical appraisal. Inplasy protocol 202090105. doi:

10.37766/inplasy2020.9.0105

Received: 29 September 2020

Published: 29 September 2020

Corresponding author:

Luísa Prada

luisarussoprada@gmail.com

Author Affiliation:

Laboratory of Clinical

Pharmacology and

Therapeutics, Faculty of

Medicine, University of Lisbon,

Lisbon, Portugal

Support: No financial support.

Review Stage at time of this submission: Data extraction.

Conflicts of interest:

The authors declare that they have no conflicts of interest.

\section{Overview of systematic reviews and meta-analyses published in Portuguese Medical journals: time trends and critical appraisal}

\author{
Prada, L1: Prada, A2; Antunes, M³; Costa, J4; Caldeira, D5.
}

Review question / Objective: To evaluate the publication trend, research fields, and overall quality of systematic reviews published in Portuguese medical journals indexed in MEDLINE.

Condition being studied: This study aims to evaluate the publication trend, the clinical research field, the typology of systematic reviews, and the overall methodological quality of systematic reviews (assessed using AMSTAR II (A Measurement Tool to Assess Systematic Reviews-II)) published in indexed Portuguese medical journals.

Information sources: Potentially eligible systematic reviews were identified through an electronic search through PubMed, targeting Portuguese Medical journals indexed in MEDLINE.

INPLASY registration number: This protocol was registered with the International Platform of Registered Systematic Review and Meta-Analysis Protocols (INPLASY) on 29 September 2020 and was last updated on 29 September 2020 (registration number INPLASY202090105).

\section{INTRODUCTION}

Review question / Objective: To evaluate the publication trend, research fields, and overall quality of systematic reviews published in Portuguese medical journals indexed in MEDLINE.

Condition being studied: This study aims to evaluate the publication trend, the clinical 
research field, the typology of systematic reviews, and the overall methodological quality of systematic reviews (assessed using AMSTAR II (A Measurement Tool to Assess Systematic Reviews-II)) published in indexed Portuguese medical journals.

\section{METHODS}

Participant or population: Portuguese medical journals indexed in MEDLINE.

Intervention: No intervention.

Comparator: No comparator.

Study designs to be included: systematic reviews and meta-analyses.

Eligibility criteria: Studies published in Portuguese medical journals indexed in MEDLINE; Studies that are systematic reviews or meta-analyses; Published in full in English or Portuguese.

Information sources: Potentially eligible systematic reviews were identified through an electronic search through PubMed, targeting Portuguese Medical journals indexed in MEDLINE.

Main outcome(s): To evaluate the publication trend, the clinical research field, the typology of systematic reviews, and the overall methodological quality of systematic reviews (assessed using AMSTAR II (A Measurement Tool to Assess Systematic Reviews-II)) published in indexed Portuguese medical journals.

Quality assessment / Risk of bias analysis: The methodological quality assessment of the systematic reviews and meta-analysis was assessed using the AMSTAR II (A Measurement Tool to Assess Systematic Reviews-II). Overall systematic reviews quality was classified as "Critically Low quality", "Low quality", "Moderate quality" and "High quality".

Strategy of data synthesis: Qualitative Data Synthesis.

Subgroup analysis: No subgroup analysis.
Sensibility analysis: No sensibility analysis.

Country(ies) involved: Portugal.

Keywords: AMSTAR II, quality, Systematic review, Meta-analysis, Portugal.

Contributions of each author:

Author 1 - Luísa Prada.

Author 2 - Ana Prada.

Author 3 - Miguel Antunes.

Author 4 - João Costa.

Author 5 - Daniel Caldeira. 